OPEN ACCESS

Edited by:

Peng Qu,

National Institutes of Health (NIH),

United States

Reviewed by:

Richard Eric Davis,

University of Texas MD Anderson

Cancer Center, United States

Panayotis Verginis,

Biomedical Research Foundation of

the Academy of Athens (BRFAA),

Greece

Jiaxin Liang,

Dana-Farber Cancer Institute, United States

*Correspondence: Lyse A. Norian Inorian@uab.edu

Specialty section:

This article was submitted to

Cancer Immunity

and Immunotherapy,

a section of the journal

Frontiers in Immunology

Received: 03 August 2020 Accepted: 15 September 2020

Published: 06 October 2020

Citation:

Gibson JT, Orlandella RM, Turbitt WJ, Behring M, Manne $U$, Sorge RE and Norian LA (2020) Obesity-Associated

Myeloid-Derived Suppressor

Cells Promote Apoptosis

of Tumor-Infiltrating CD8 T

Cells and Immunotherapy

Resistance in Breast Cancer.

Front. Immunol. 11:590794.

doi: 10.3389/fimmu.2020.590794

\section{Obesity-Associated Myeloid-Derived Suppressor Cells Promote Apoptosis of Tumor-Infiltrating CD8 T Cells and Immunotherapy Resistance in Breast Cancer}

Justin T. Gibson ${ }^{1}$, Rachael M. Orlandella ${ }^{1}$, William J. Turbitt ${ }^{2}$, Michael Behring ${ }^{3}$, Upender Manne ${ }^{3,4}$, Robert E. Sorge ${ }^{5,6}$ and Lyse A. Norian ${ }^{2,4,6 *}$

1 Graduate Biomedical Sciences, University of Alabama at Birmingham, Birmingham, AL, United States, ${ }^{2}$ Department of Nutrition Sciences, University of Alabama at Birmingham, Birmingham, AL, United States, ${ }^{3}$ Department of Pathology, University of Alabama at Birmingham, Birmingham, AL, United States, ${ }^{4}$ O'Neal Comprehensive Cancer Center, University of Alabama at Birmingham, Birmingham, AL, United States, ${ }^{5}$ Department of Psychology, University of Alabama at Birmingham, Birmingham, AL, United States, ${ }^{6}$ Nutrition Obesity Research Center, University of Alabama at Birmingham, Birmingham, AL, United States

Nearly $70 \%$ of adults in the US are currently overweight or obese. Despite such high prevalence, the impact of obesity on antitumor immunity and immunotherapy outcomes remains incompletely understood, particularly in patients with breast cancer. Here, we addressed these gaps in knowledge using two murine models of breast cancer combined with diet-induced obesity. We report that obesity increases CXCL1 concentrations in the mammary tumor microenvironment, driving CXCR2-mediated chemotaxis and accumulation of granulocytic myeloid-derived suppressor cells (G-MDSCs) expressing Fas ligand (FasL). Obesity simultaneously promotes hyperactivation of CD8 tumorinfiltrating lymphocytes (TILS), as evidenced by increased expression of CD44, PD-1, $\mathrm{Ki}-67$, IFN $\gamma$, and the death receptor Fas. Accordingly, G-MDSCs induce Fas/FasLmediated apoptosis of CD8 T cells ex vivo and in vivo. These changes promote immunotherapy resistance in obese mice. Disruption of CXCR2-mediated G-MDSC chemotaxis in obese mice is sufficient to limit intratumoral G-MDSC accumulation and improve immunotherapy outcomes. The translational relevance of our findings is demonstrated by transcriptomic analyses of human breast tumor tissues, which reveal positive associations between CXCL1 expression and body mass index, poor survival, and a MDSC gene signature. Further, this MDSC gene signature is positively associated with FASLG expression. Thus, we have identified a pathway wherein obesity leads to increased intratumoral CXCL1 concentrations, which promotes CXCR2-mediated accumulation of FasL ${ }^{+}$G-MDSCs, resulting in heightened CD8 TIL apoptosis and immunotherapy resistance. Disruption of this pathway may improve immunotherapy outcomes in patients with breast cancer and obesity.

Keywords: myeloid-derived suppressor cells (MDSCs), obesity, immunotherapy resistance, breast cancer, tumorinfiltrating lymphocyte apoptosis, Fas ligand (FasL) 


\section{INTRODUCTION}

Obesity is a major health epidemic in the US, affecting nearly $40 \%$ of adults (1). Excess body weight increases the incidence of 13 types of cancer (2), promotes disease progression $(3,4)$, impairs antitumor immunity $(5,6)$, promotes resistance to targeted and chemotherapies $(7,8)$, and worsens survival for cancer patients $(9,10)$, especially those with breast cancer. However, the impact of obesity on immunotherapy outcomes in patients with breast cancer is unknown.

In 2019, the FDA granted accelerated approval for the treatment of select triple negative breast cancer patients with an immunotherapy/chemotherapy combination based on results reported from the phase III IMpassion130 clinical trial (11). However, despite immunotherapeutics dramatically improving outcomes for a subset of patients with cancer, many still do not respond. As such, research efforts are concentrated on identifying and targeting factors underlying immunotherapeutic resistance. Obesity may be one such factor. Recent findings from a phase I clinical trial of patients with breast cancer treated with atezolizumab (anti-PD-L1) indicate that elevated baseline plasma levels of interleukin 6 (IL-6) and C-reactive protein (CRP) are associated with reduced progression free and overall survival (12). As both IL-6 and CRP are biomarkers of obesity-associated inflammation, this raises the possibility that obesity may impair immunotherapeutic efficacy in patients with breast cancer. In support of this idea, obesity was previously shown to be associated with impaired responses to targeted and chemotherapies in patients with breast cancer $(7,8)$. However, given the short duration of its clinical use, the impact of obesity on immunotherapy outcomes in patients with breast cancer has not yet been investigated.

Molecular markers of obesity (e.g. IL-6, CRP, leptin, interleukin 1 beta [IL-1 $\beta$ ]) increase the generation of myeloid-derived suppressor cells (MDSCs) (13-16). MDSCs are a heterogeneous immunosuppressive population of cells that function to inhibit protective antitumor immunity to promote tumor growth and progression. To date, only one study has investigated the impact of obesity-associated MDSCs in mediating mammary tumor growth (14). However, this study did not assess the impact of MDSCs on response to immunotherapy. Given that intratumoral accumulation of MDSCs has been identified as a mechanism of immunotherapy resistance $(17,18)$, we asked if obesity would enhance MDSC-mediated immunotherapy resistance in a preclinical model of breast cancer.

\section{MATERIALS AND METHODS}

\section{Research Resource Identifiers}

Research Resource Identifiers (RRIDs) and other detailed identifier information (vendor, clone, catalog number etc.) for antibodies and key reagents used in this study can be found in this text and Supplementary Table 1.

\section{Murine Models of Obesity}

Wildtype C57BL/6 female mice were purchased from Charles River at 7-8 weeks of age. Following one week of acclimation, animals were randomized to a NIH31 formulated low-fat diet (LFD; $14 \% \mathrm{kcal}$ from fat, LabDiet), standard American diet (SAD; 36\% kcal from fat, Envigo), or high-fat diet (HFD; 60\% kcal from fat, Research Diets) for 16 weeks, thereby generating lean (LFD) or diet-induced obese (SAD and HFD) mice. All animal experiments were conducted as approved by the Animal Resources Program and Institutional Animal Care and Use Committee at the University of Alabama at Birmingham (UAB).

\section{In Vivo Tumor Modeling}

The E0771 murine mammary tumor cell line was purchased from CH3 BioSystems (RRID: CVCL_GR23) and engineered to express firefly luciferase (E0771-fluc) via transduction with lentiviral particles (Kerafast) according to the manufacturer's protocol. Cells were maintained in RPMI1640 complete media supplemented with $10 \%$ FBS, $10 \mathrm{mM}$ HEPES, 100 units $/ \mathrm{ml}$ Penicillin, and $100 \mu \mathrm{g} / \mathrm{ml}$ Streptomycin. The Py8119 murine mammary tumor cell line was purchased from ATCC (RRID : CVCL_AQ09) and cells were maintained in F-12K complete media supplemented with 5\% FBS. Neither cell line was authenticated after purchase and both were determined to be free of mycoplasma contamination.

Mice were injected with 2.5e5 E0771-fluc or 1e6 Py8119 cells subcutaneously into mammary fat pad number four. Tumor outgrowth was monitored using a dial caliper and bioluminescence imaging. To measure E0771-fluc tumor bioluminescence, tumorbearing mice were injected intraperitoneally with $1 \mathrm{mg}$ of luciferin, anesthetized, and imaged using an IVIS Lumina III imaging system and the Living Image Software Version 4.7.2 (Perkin Elmer).

\section{NanoString and Gene Ontology Analysis of Tumor Immune Microenvironment}

At day 28 experimental endpoint, E0771-fluc tumors were excised and a portion was preserved in RNAlater. RNA was batch isolated and submitted to the UAB nanoString facility for interrogation using the nCounter PanCancer Immune Profiling Panel (XT-CSO-MIP1-12) according to the manufacturer's instructions. Data analysis was performed using nCounter Analysis Software (Version 4.0) with the Advanced Analysis (Version 2.0) plugin. Differentially expressed genes were identified as those with a $p$ value $<0.05$ and fold change $> \pm$ 1.5. Due to the exploratory nature of our study and intent for validation, we used raw $p$ values and did not adjust $p$ values for multiple comparisons.

The nanoString tumor immunogenetic profiling dataset expression matrix from $\mathrm{n}=72$ subjects with triple negative breast cancer (TNBC) was obtained from a previously published manuscript (17). Body mass index (BMI) information for this cohort of subjects was kindly provided by Dr. Xiang "Shawn" Zhang, Baylor College of Medicine. Of the 72 patient tissues evaluated, 4 were excluded from analysis due to lack of BMI data, being underweight, or issues with sample quality control. The final cohort for analysis consisted of $n=68$ samples. Linear regression analyses were performed for BMI versus $\log 2$ expression of each of the 730 genes. Due to the exploratory nature of the study, those with a $\mathrm{p}<0.1$ were investigated further. Of the 730 genes, 73 were associated with BMI; 8 positively and 65 negatively. 
From our murine study, differentially expressed genes were separated into groups of upregulated or downregulated genes and subjected to Gene Ontology enrichment analysis using the PANTHER classification system to identify associated biological processes (http://geneontology.org/) (19). This was also done for the clinical analyses using genes positively associated with BMI, identified as described above.

\section{Flow Cytometry}

Spleen and tumor tissues were excised and mechanically homogenized using the Miltenyi gentleMACS Dissociator, followed by enzymatic digestion in $5 \mu \mathrm{g} / \mathrm{ml}$ Liberase TM and $37.5 \mu \mathrm{g} / \mathrm{ml} \mathrm{DNase} \mathrm{I} \mathrm{at} 37^{\circ} \mathrm{C}$ with gentle agitation for 15 or $30 \mathrm{~min}$, respectively. Resulting homogenates were filtered (70um) to obtain single cell suspensions. Cells were stained with fixable Zombie Aqua viability dye, Fc blocked, and incubated with fluorophore-conjugated antibodies to surface markers. Evaluation of intracellular markers was conducted using BD's Cytofix/Cytoperm kit according to manufacturer's protocol. For evaluation of T cell cytokine production, single cell tumor homogenates were stimulated with anti-CD3 $(8 \mu \mathrm{g} / \mathrm{ml})$ and anti-CD28 $(10 \mu \mathrm{g} / \mathrm{ml})$ for $4 \mathrm{~h}$ with the addition of GolgiPlug during the last $2 \mathrm{~h}$. Cells were then harvested and stained as indicated above for surface and intracellular markers. Apoptosis was evaluated using the BD Annexin V Apoptosis Detection Kit according to manufacturer's protocol.

Samples were analyzed on an Attune NxT cytometer after compensation using BD Comp Beads. Analyses were conducted using FlowJo (Version 10) and all gates were objectively drawn based on fluorescence minus one (FMO) controls for the tissue of interest. Detailed information for the antibodies used in this study is provided in Supplementary Table 1.

\section{Quantification of Soluble Proteins}

Tumors were excised and placed in Dulbecco's Phosphate Buffered Saline (DPBS; 0.2g tissue per $1 \mathrm{ml}$ DPBS) followed by mechanical homogenization using a Miltenyi gentleMACS Dissociator. Tumor homogenates were centrifuged and supernatants were collected and stored for batch analysis of soluble proteins using the Bio-Plex Multiplex Immunoassay (Bio-Rad) according to the manufacturer's protocols.

\section{In Vivo Anti-CD8 $\beta$ Antibody-Mediated CD8 T Cell Depletion}

On days $1,3,5,7,14,21$, and 27 post tumor challenge, lean and $\mathrm{DIO}$ animals received an intraperitoneal injection containing $100 \mu \mathrm{g}$ of anti-CD8 $\beta$ or isotype control antibody (Bio X Cell). Animals were euthanized on day 28 post tumor challenge, at which time tumor tissues were excised and CD8 TILs were evaluated by flow cytometry.

\section{In Vivo Anti-Gr-1 Antibody-Mediated MDSC Depletion}

On day 19 post tumor challenge, DIO animals received an intratumoral injection containing $200 \mu \mathrm{g}$ of anti-Gr-1 or isotype control antibody (Bio X Cell). On days 20, 21, and 22 post tumor challenge, animals received a follow-up injection containing $100 \mu \mathrm{g}$ of their respective antibody. Animals were euthanized on day 23 post tumor challenge, at which time tumor tissues were excised and infiltrating leukocytes were evaluated by flow cytometry.

\section{G-MDSC Induction of Apoptosis}

$\mathrm{CD}^{+} \mathrm{T}$ cells were positively selected from spleens of naive 8- to 12-week-old C57BL/6 female mice using Miltenyi MACS Enrichment according to the manufacturer's protocol. Enriched $\mathrm{CD}^{+} \mathrm{T}$ cells were activated in vitro for $72 \mathrm{~h}$ using $1 \mu \mathrm{g} / \mathrm{ml}$ antiCD3 and $1 \mu \mathrm{g} / \mathrm{ml}$ anti-CD28 to induce Fas expression. Tumorinfiltrating G-MDSCs from DIO mice were sort-purified on a BD FACSMelody after being pre-enriched using anti-CD11b MACS beads from Miltenyi, as per the manufacturer's protocol. Prior to co-culture, purified G-MDSCs were incubated in the presence or absence of $50 \mu \mathrm{g} / \mathrm{ml}$ anti-FasL neutralizing antibody for $1 \mathrm{~h}$. In vitro activated $\mathrm{CD}^{+} \mathrm{T}$ cells were then co-cultured with G-MDSCs in the continued presence or absence of anti-FasL for $24 \mathrm{~h}$. Cells were harvested and stained for flow cytometry to evaluate CD8 T cell apoptosis as indicated above.

\section{Immunotherapy}

Mice with established tumors within a $25-100 \mathrm{~mm}^{2}$ range $(\sim 50$ $\mathrm{mm}^{2}$ on average) were randomized to receive saline or immunotherapy. Immunotherapy consisted of $1 \times 10^{9} \mathrm{PFU}$ of adenovirus (Ad) encoding murine TNF-related apoptosis inducing ligand (TRAIL; AdT) and $50 \mu \mathrm{g}$ of the TLR9 agonist CpG 1826 oligodeoxynucleotide (AdT+CpG) (20). Saline or AdT+CpG were administered in 50uL volumes via intratumoral injection.

Disruption of CXCR2 signaling was accomplished using the CXCR2 antagonist SB 225002 (Tocris Bioscience). Beginning 2 days prior to tumor challenge and every 2 days after until day 26 , $5 \mathrm{mg} / \mathrm{kg}$ of CXCR2a was administered intraperitoneally to lean and DIO animals.

\section{Clinical Outcomes}

Kaplan-Meier Plotter (KM Plotter; https://kmplot.com/) was used to evaluate the association of CXCL1 expression and overall survival in systemically untreated patients with basal breast cancer (21). The following parameters were used for analysis with regard to CXCL1: auto select best cutoff, overall survival, intrinsic subtype: basal, and systemically untreated patients. Log rank $\mathrm{p}$ value and hazard ratio (HR) were calculated as part of the analysis.

\section{Clinical Transcriptomic Analyses}

Transcriptomic data were downloaded from The Cancer Genome Atlas (TCGA) using the curatedTCGAData package (https://bioconductor.org/packages/release/data/experiment/ $\mathrm{html} /$ curatedTCGAData.html). The most recent version of TCGA RNASeq data (title "BRCA_RNASeq2GeneNorm20160128”) was used for analysis. To approximate expression values, RNASeq 2 count data were $\log 2$ transformed and the mean expression of a previously published multi-gene MDSC signature (22) was calculated. The MDSC expression score was then separated into tertiles and compared to log2 expression values for genes of interest in tumor tissues from $n=88$ patients with basal breast cancer. 


\section{Statistical Analysis}

Statistical analyses were performed using GraphPad Prism Version 7.0. Gaussian distribution was assessed using the Shapiro-Wilk normality test. Comparisons of two groups was accomplished using non-parametric Mann-Whitney $U$ tests or parametric two-tailed unpaired t-tests as appropriate. Comparisons of three groups was accomplished using nonparametric Kruskal-Wallis tests with Dunn's multiple comparisons tests or parametric one-way ANOVAs with Tukey's or Dunnet's multiple comparisons tests as appropriate. Analysis of four groups was carried out using non-parametric Kruskal-Wallis tests or parametric one-way ANOVAs with post-hoc pairwise comparisons of group means of interest using non-parametric Mann-Whitney U tests, parametric two-tailed unpaired t-tests, or Tukey's multiple comparisons test, as appropriate. Differences in tumor growth over time were assessed using repeated measures two-way ANOVAs with Tukey's multiple comparisons test. Associations between clinical gene expression and BMI were evaluated using linear regression analyses. Statistical differences are denoted throughout as ${ }^{*} \mathrm{p}<0.05,{ }^{* *} \mathrm{p}<0.01$, ${ }^{* *} \mathrm{p}<0.001$, ${ }^{* * * *} \mathrm{p}<0.0001$. Non-significant (ns) $\mathrm{p}$ values $\leq 0.15$ are indicated throughout.

\section{RESULTS}

\section{Obesity Promotes Mammary Tumor Growth and Remodeling of the Tumor Immune Microenvironment}

To evaluate the impact of obesity on mammary tumor growth, we utilized two models of diet-induced obesity (DIO) wherein animals were randomized to either a standard American diet (SAD; 36\% kcal from fat) or high-fat diet (HFD; 60\% kcal from fat) for 16 weeks. Low-fat diet-fed (LFD; $14 \% \mathrm{kcal}$ from fat) animals served as age-matched lean controls. SAD- and HFDfeeding led to significantly increased body weights relative to LFD-fed lean controls, with HFD-fed animals having further increases in body weights relative to SAD-fed animals (Figure 1A). Lean and DIO mice were then challenged with E0771 mammary tumor cells expressing firefly luciferase (E0771-fluc) and tumor growth was monitored via caliper measurements, bioluminescent imaging, and excised tumor weights. All methods indicated that both SAD- and HFD-induced obesity promote accelerated mammary tumor growth (Figures 1B-D).

We next asked how DIO impacted the composition of the tumor immune microenvironment. To this end, we evaluated the immunogenetic profile of tumors from LFD- and HFD-fed animals using nanoString to screen for genes of interest. Doing so revealed differential expression (DE) of 86 of the 730 genes surveyed (Figure 1E and Supplementary Table 2). Gene Ontology (GO) assessment of biological processes involved with downregulated genes largely identified $\mathrm{T}$ cell alterations, whereas upregulated genes were associated with neutrophil/ granulocyte trafficking and apoptotic signaling (Figure 1F). Flow cytometric analysis confirmed obesity-associated reductions in CD8 tumor-infiltrating lymphocytes (TILs) and increases in myeloid-derived suppressor cells (MDSCs), a subset of which are phenotypically synonymous with tumor-associated neutrophils (Figure 1G). Prior studies have shown that these immunologic alterations are associated with negative clinical outcomes in patients with breast cancer, independent of obesity status $(23,24)$. Most notably, as body weights increased $(\mathrm{LFD}<\mathrm{SAD}<\mathrm{HFD})$, there were corresponding increases in tumor burden and MDSC abundance, whereas CD8 TIL abundance decreased. Similar obesity-associated alterations to tumor growth and leukocyte composition were also seen in the Py8119 mammary tumor model, indicating that these results were not specific to a single tumor model (Supplementary Figure 1).

We next wanted to explore whether a causal relationship existed between the abundance of CD8 TILs and mammary tumor growth in LFD-fed lean and HFD-fed DIO mice. Following depletion of CD8 $\mathrm{T}$ cells, lean mice experienced accelerated tumor growth as expected, demonstrating the critical role of CD8 TILs in mediating antitumor immunity in the E0771-fluc tumor model (Supplementary Figures 2A, B). Unsurprisingly, depletion of CD8 T cells in DIO mice did not impact mammary tumor growth (Supplementary Figure 2A). Quantification of CD8 TILs suggested that this was likely due to the already extremely low levels of CD8 TILs present in the obese tumor microenvironment (Supplementary Figure 2B). Thus these data suggested that obesity-associated reductions in CD8 TIL abundance impair antitumor immunity.

\section{Obesity Promotes CD8 TIL Hyperactivation and Apoptosis in the Mammary Tumor Microenvironment}

Given that obesity was associated with reductions in total CD8 TILs, we further sought to identify potential alterations in their phenotype and mechanisms underlying their reduction. Surprisingly, the frequency of CD8 TILs with an effector phenotype increased with obesity while naive CD8 TILs decreased (Figure 2A). Accordingly, increases were seen in the percentages of $\mathrm{PD}-1^{+}, \mathrm{Ki}-67^{+}$, and IFN $\gamma^{+}$ CD8 TILs, consistent with increased antigen engagement, proliferation, and cytokine production with obesity (Figures 2 BD). Notably, regardless of phenotype, total effector and naive CD8 TIL subsets and intratumoral IFN $\gamma$ concentration were decreased with obesity (Supplementary Figure 3). Our immunogenetic profiling results indicated that obesity was associated with increased apoptotic signaling (Figure 1F). Thus, we next asked if obesity-associated TIL hyperactivation promoted apoptosis, causing decreased CD8 TIL abundance (Figure 1G). Annexin V staining revealed that obesity was indeed associated with increased percentages of apoptotic CD8 TILs (Figure 2E). Further, DIO animals had reduced percentages of CD8 TILs expressing antiapoptotic Bcl-2 (Figure 2F) and increased percentages of CD8 TILs expressing the death receptor Fas (Figure 2G). These results corroborated our immunogenetic profiling results (Figures 1E, F) and indicated that CD8 TILs were hyperactivated and poised for apoptosis with obesity.

Having established that a higher percentage of CD8 TILs from DIO animals were expressing Fas and undergoing apoptosis, we 


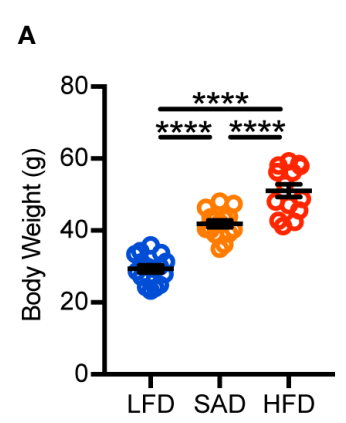

$\mathbf{E}$

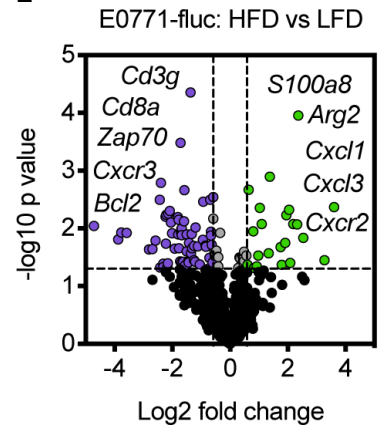

B

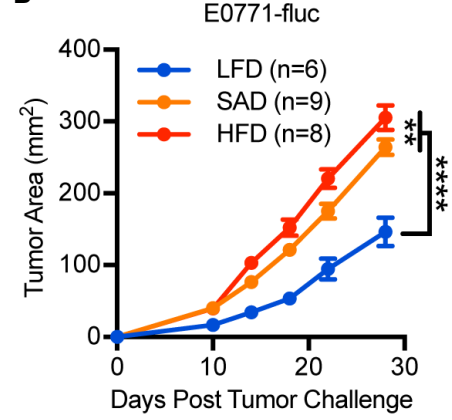

C

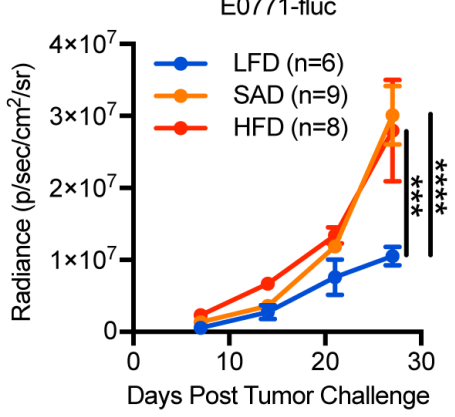

D

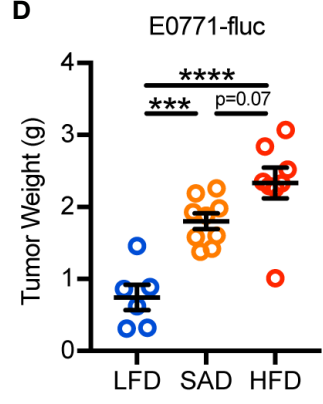

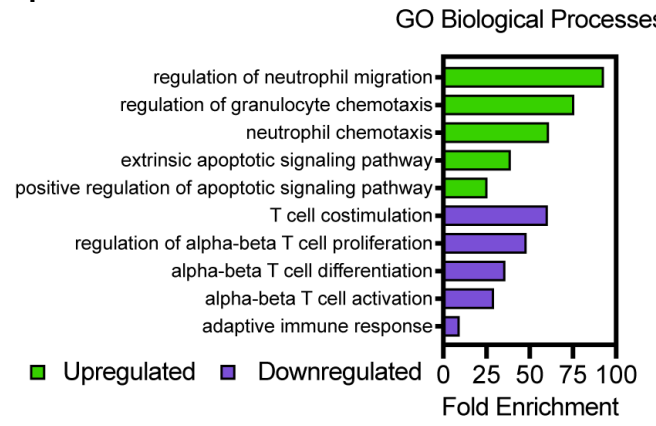

G

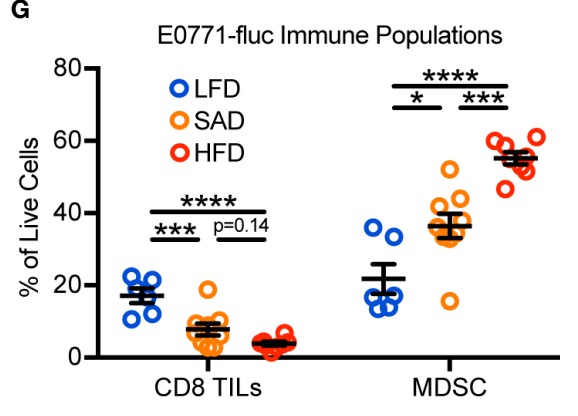

FIGURE 1 | Diet-induced obesity promotes mammary tumor growth and remodeling of the tumor immune microenvironment. (A) Body weight of animals 16 weeks after randomization to low-fat diet (LFD; 14\% kcal from fat), standard American diet (SAD; 36\% kcal from fat), or high-fat diet (HFD; 60\% kcal from fat). Following diet randomization, animals were challenged with E0771-fluc mammary tumor cells and tumor growth monitored via (B) caliper measurements, (C) bioluminescent imaging of firefly luciferase expressing E0771 tumor cells (E0771-fluc), and (D) excised tumor weights. (E) Volcano plot showing differentially expressed (DE) genes from tumors of HFD-fed animals vs LFD-fed animals. Dotted lines indicate $p=0.05$ and fold change $= \pm 1.5$. (F) Gene Ontology (GO) enrichment analysis for biological processes associated with downregulated (purple) and upregulated (green) DE genes from (E). (G) Flow cytometric analysis of day 28 excised mammary tumors for $\mathrm{CD}^{+} \mathrm{CD}^{+}$tumor-infiltrating lymphocytes and CD11 ${ }^{+} \mathrm{Gr}-1^{+}$MDSCs. Data are pooled from multiple independent experiments and presented as means \pm SEM. Statistical differences were calculated using parametric one-way ANOVAs or repeated measures two-way ANOVAs, both with Tukey's multiple comparisons tests $\left({ }^{*} p<0.05,{ }^{* *} p<0.01,{ }^{\star \star *} p<0.001,{ }^{\star \star \star *} p<0.0001\right)$.

next asked what sources of FasL existed within the tumor microenvironment that could potentially promote Fas/FasLmediated apoptosis of CD8 TILs. In both lean and DIO animals, $\mathrm{CD}_{1} 1 \mathrm{~b}^{+} \mathrm{Gr}-1^{+}$MDSCs were the largest contributors of FasL expression (Figure 2H). Notably, obesity did not impact the apoptotic phenotype of splenic CD8 T cells where $\mathrm{FasL}^{+}$MDSCs were minimally abundant (Figures 2I, J). These results suggested that the abundance of $\mathrm{FasL}^{+}$MDSCs was associated with CD8 TIL apoptosis.

\section{Obesity Promotes the Recruitment and Accumulation of FasL ${ }^{+}$G-MDSCs Which Induce Fas/FasL-Mediated Apoptosis of Hyperactivated CD8 TILs}

We next sought to identify the mechanism(s) by which obesity promoted MDSC accumulation in murine mammary tumors. Murine MDSCs exist as two main sub-types: monocytic MDSCs (M-MDSCs) and granulocytic MDSCs (G-MDSCs). With obesity, only the G-MDSC subset was increased within tumors (Figure 3A). Our tumor immunogenetic profiling results revealed that obesity significantly increased the expression of Il1b, Cxcl1, Cxcl3, S100a8, and Csf3. These genes encode cytokines and chemokines responsible for the generation and trafficking of G-MDSCs, providing evidence for enhanced MDSC generation and chemotaxis with obesity (Figure 3B). Intratumoral protein concentrations of CXCL1 were further found to be elevated in the obese tumor microenvironment (Figure 3C). CXCL1 and CXCL3 are chemokines that promote G-MDSC chemotaxis via their cognate receptor CXCR2 (25). CXCR2 was highly and equivalently expressed on tumorinfiltrating G-MDSCs from both lean and obese animals (Figure 3D). Thus, these results suggest that obesity enhances generation and CXCL1/CXCR2-mediated G-MDSC chemotaxis.

We next asked how obesity impacted FasL expression by GMDSCs and whether MDSCs were, in fact, responsible for CD8 TIL apoptosis. Approximately $90 \%$ of G-MDSCs from lean and DIO animals expressed FasL (Figure 3E). Interestingly, although the frequency of G-MDSCs expressing FasL was unchanged, the per cell expression of FasL was elevated with obesity (Figure 3F). We next sought to confirm that $\mathrm{FasL}^{+}$G-MDSCs could directly induce Fas/FasL-mediated apoptosis of CD8 T cells. Splenic CD8 
A
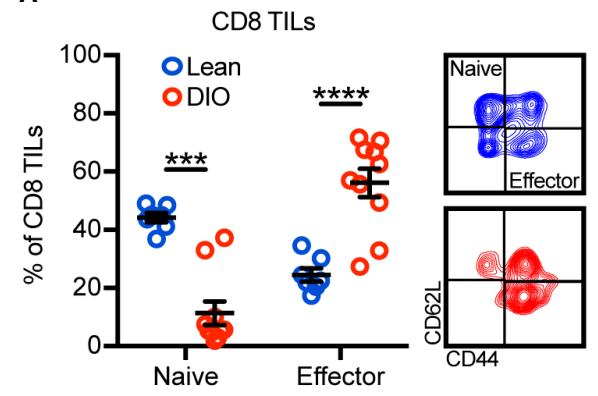

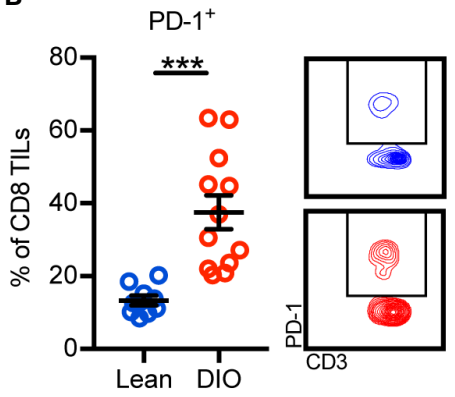

D

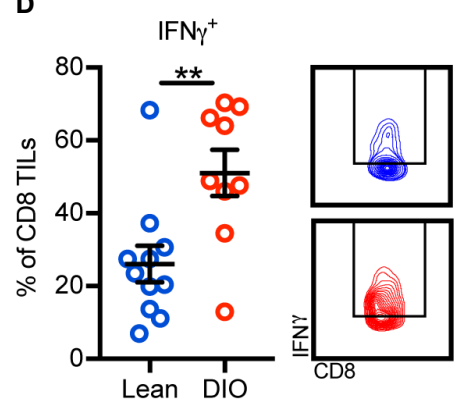

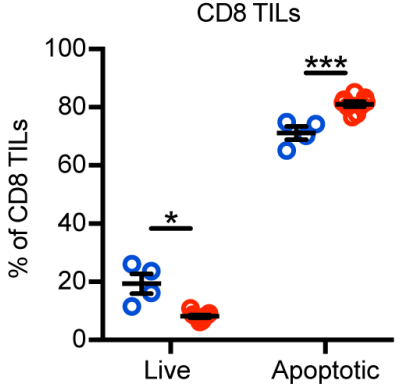

C

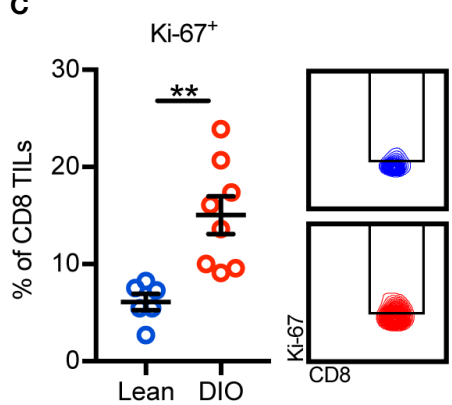

G

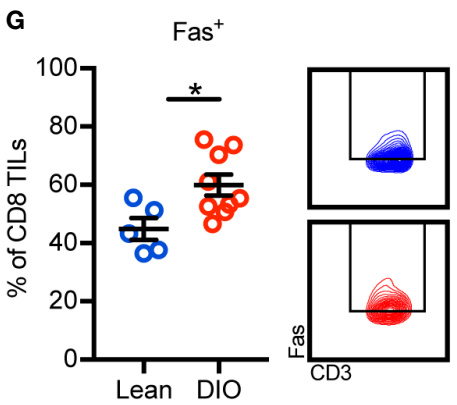

H

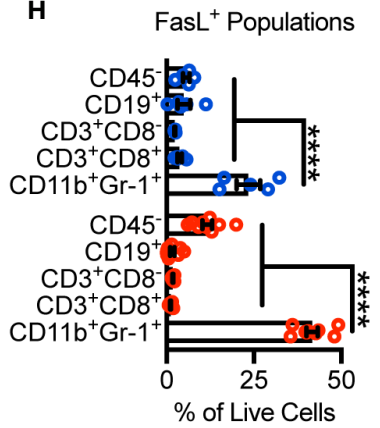

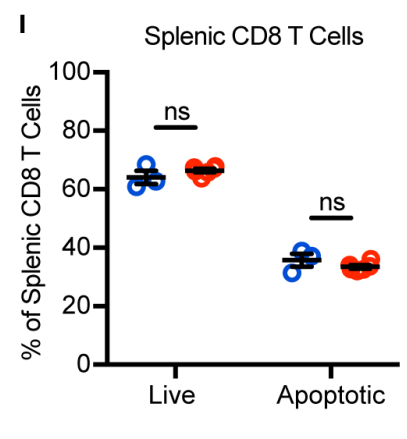

$\mathbf{F}$

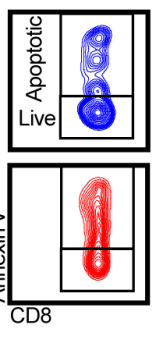

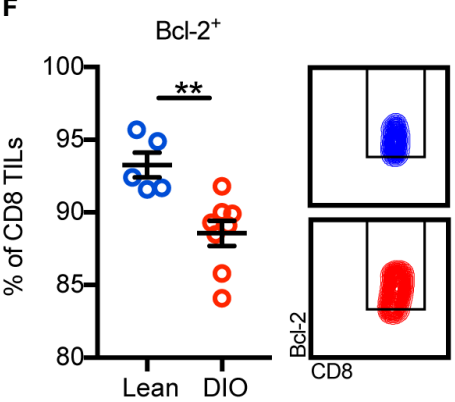

FIGURE 2 | Obesity promotes CD8 TIL hyperactivation and apoptosis in the mammary tumor microenvironment. Flow cytometric analysis of day 28 excised E0771fluc mammary tumors for (A) naive (CD44-CD62 ${ }^{+}$) and effector (CD44 ${ }^{+}$CD62 L ) CD8 TILs. Expression of (B) PD-1, (C) Ki-67, and (D) IFN $\gamma$ by CD8 TILs.

(E) Quantification of live (annexin $V^{-}$) and apoptotic (annexin $V^{+}$) CD8 TILs. Expression of (F) Bcl-2 and (G) Fas by CD8 TILs. (H) Quantification of FasL ${ }^{+}$tumor infiltrating populations. (I) Quantification of live (annexin $V^{-}$) and apoptotic (annexin $V^{+}$) splenic CD8 T cells and (J) FasL ${ }^{+}$splenic populations. (A-D, F) Data are pooled from multiple independent experiments or (E, G-J) are from a single representative experiment and presented as means \pm SEM. Statistical differences were calculated using parametric t-tests or non-parametric Mann-Whitney U tests as appropriate and parametric one-way ANOVAs with Dunnet's multiple comparisons tests $\left({ }^{\star} \mathrm{p}<0.05,{ }^{\star \star} \mathrm{p}<0.01,{ }^{\star \star *} \mathrm{p}<0.001,{ }^{\star \star \star \star} \mathrm{p}<0.0001\right.$, ns $=$ non-significant).

T cells were activated in vitro to induce expression of Fas then cocultured with G-MDSCs sort-purified from the tumors of obese mice in the presence or absence of FasL neutralization. FasL neutralization demonstrated that G-MDSCs were not only capable of inducing CD8 T cell apoptosis, but that they did so in a largely FasL-dependent manner (Figure 3G). We also asked whether G-MDSC-induced CD8 TIL apoptosis occurred in vivo. Short-term anti-Gr-1-mediated MDSC depletion in DIO animals resulted in decreased percentages of apoptotic CD8 TILs relative to isotype-treated controls (Figures $\mathbf{3 H}, \mathbf{I}$ ). Thus, these data demonstrate that obesity promotes the recruitment and intratumoral accumulation of $\mathrm{FasL}^{+}$G-MDSCs that functionally suppress hyperactivated $\mathrm{Fas}^{+}$CD8 TILs via the induction of apoptosis.

\section{Obesity Promotes Immunotherapy Resistance and Is Associated With G-MDSC Accumulation}

Our findings that obesity negatively impacted antitumor immunity via enhanced G-MDSC accumulation and CD8 TIL apoptosis prompted us to ask if these changes impaired immunotherapy outcomes. Because obesity accelerates tumor growth (Figure 1), we first normalized tumor areas at treatment initiation so that tumor burdens were equivalent across groups 
A

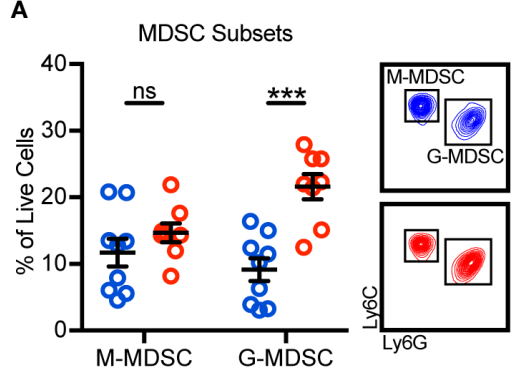

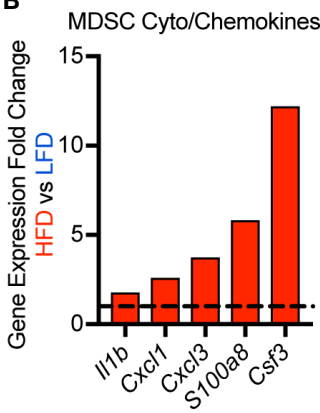

C

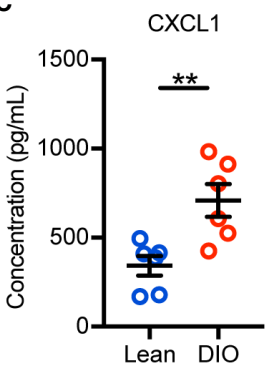

D

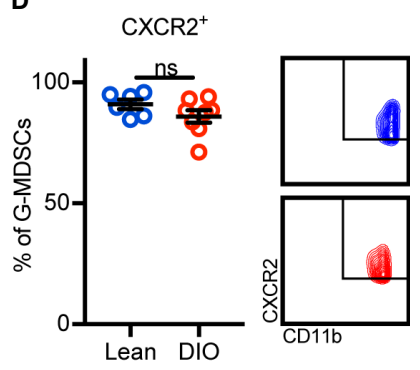

E

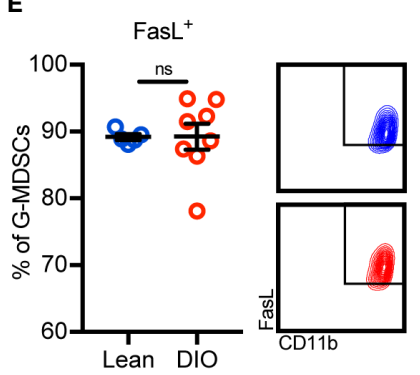

$\mathbf{F}$

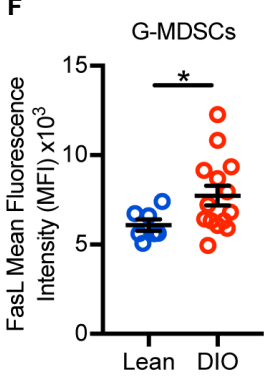

G

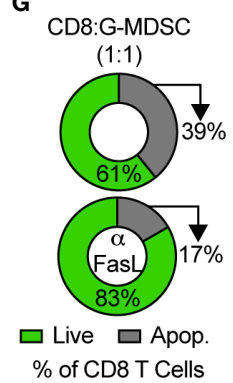

H

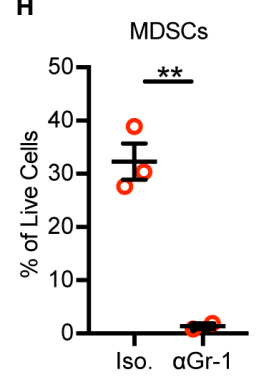

I

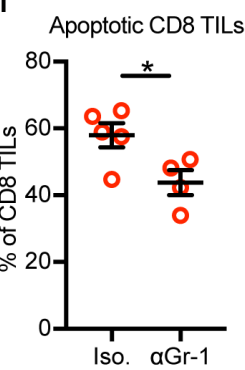

FIGURE 3 | Obesity promotes the recruitment and accumulation of FasL ${ }^{+}$granulocytic myeloid-derived suppressor cells (G-MDSCs) which induce Fas/FasLmediated apoptosis of CD8 TILs. Flow cytometric analysis of day 28 excised E0771-fluc mammary tumors for (A) monocytic MDSC (M-MDSCs)

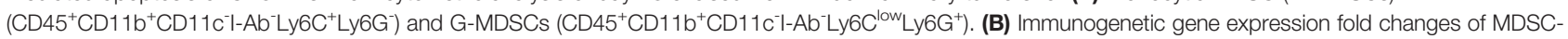
related cyto/chemokines in the tumors of HFD- vs LFD-fed animals. (C) Concentration of CXCL1 in the tumor microenvironment. Percentage of G-MDSCs expressing (D) CXCR2 and (E) FasL. (F) FasL mean fluorescence intensity of G-MDSCs. (G) Percentage of live (annexin $V^{-}$) and apoptotic (annexin $V^{+}$) in vitro activated splenic CD8 T cells after $24 \mathrm{~h}$ of co-culture with sort-purified tumor-infiltrating G-MDSCs from obese mice in the (top) absence or (bottom) presence of FasL neutralizing antibody. DIO animals were treated with intratumoral anti-Gr-1 or isotype control antibody. Quantification of intratumoral (H) MDSCs and (I) apoptotic (annexin $\mathrm{V}^{+}$) CD8 TILs. Data are pooled from (A-F, I) multiple independent experiments or are from (G, $\left.\mathbf{H}\right)$ a single representative experiment and presented as means \pm SEM. Statistical differences were calculated using parametric t-tests or non-parametric Mann-Whitney $U$ tests as appropriate $\left({ }^{*} p<0.05\right.$, ${ }^{* *} \mathrm{p}<0.01,{ }^{* \star *} \mathrm{p}<0.001, \mathrm{~ns}=$ non-significant).

and thus would not be a confounding variable in comparing outcomes in lean and DIO animals (Figure 4A). When tumors were 25-100 $\mathrm{mm}^{2}$ in area, lean and DIO animals were randomized in parallel to receive an intratumoral injection of saline or immunotherapy consisting of non-replicative adenovirus (Ad) encoding murine TNF-related apoptosis inducing ligand (TRAIL; AdT) co-administered with $\mathrm{CpG}$ oligodeoxynucleotide 1826 (AdT+CpG) (20). AdT induces tumor cell death, increasing tumor antigen availability, while CpG induces antigen-presenting cell activation and maturation (20). Collectively this therapy promotes the priming of endogenous CD8 T cells that subsequently mediate tumor control (20). Additionally, $\mathrm{CpG}$ administration induces MDSCs to mature into macrophages and dendritic cells, relieving immunosuppression (26). E0771-fluc cells express both the coxsackievirus and adenovirus receptor (CAR) that permits viral binding and the TRAIL receptor (TRAIL-R2) that induces tumor cell death (Supplementary Figure 4). Notably, normalization of tumor burden at immunotherapy administration resulted in equivalent outgrowth of tumors in lean and DIO animals treated with saline (Figure 4B direct comparison not shown, $\mathrm{p}=0.30$ ). In lean mice, $\mathrm{AdT}+\mathrm{CpG}$ immunotherapy significantly delayed tumor outgrowth, whereas in DIO animals, tumor growth progressed unchecked, demonstrating immunotherapy resistance (Figure 4B).

To discern underlying causes of $\mathrm{AdT}+\mathrm{CpG}$ immunotherapy resistance in obese mice, we examined immune responses after therapy administration. In this setting, both lean and DIO animals had statistically equivalent increases in total effector and IFN $\gamma^{+}$CD8 TILs (Figures 4C, D). However, following therapy administration, lean mice displayed reductions in CD8 TIL apoptosis and G-MDSC accumulation, whereas neither parameter changed in DIO animals (Figures 4E, F). Tumor expression levels of Cxcl1 followed a similar trend (Figure 4G), suggesting that G-MDSC recruitment persisted even after therapy administration in DIO mice. Therefore, although DIO mice mounted CD8 TIL responses equivalent to those of lean animals in response to immunotherapy, high levels of CD8 TIL apoptosis, G-MDSC accumulation, and Cxcl1 expression remained, corresponding with immunotherapy resistance.

\section{Disruption of G-MDSC Accumulation Sensitizes Obese Animals to Immunotherapy}

To determine if disruption of G-MDSC accumulation would improve immunotherapy response in lean animals and overcome 
A

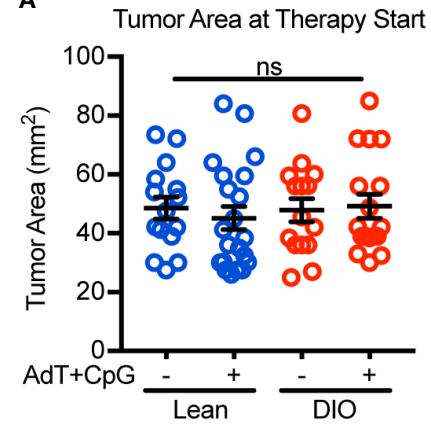

D

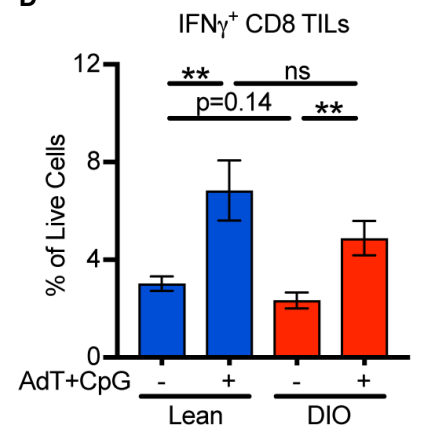

B
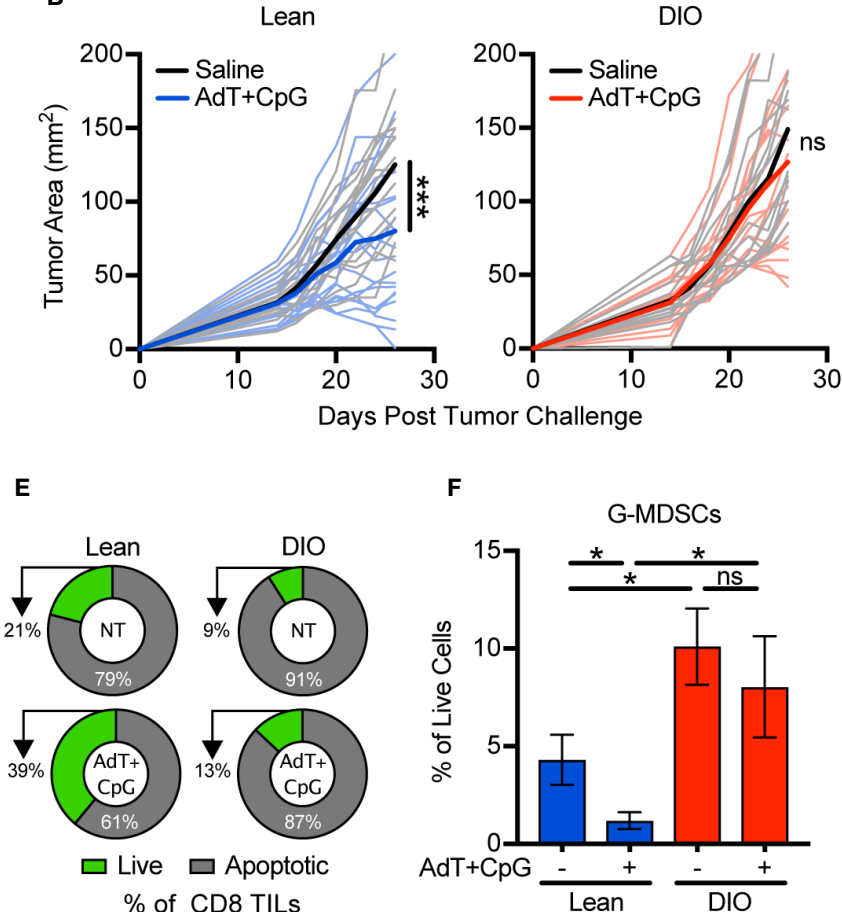

$\mathbf{F}$

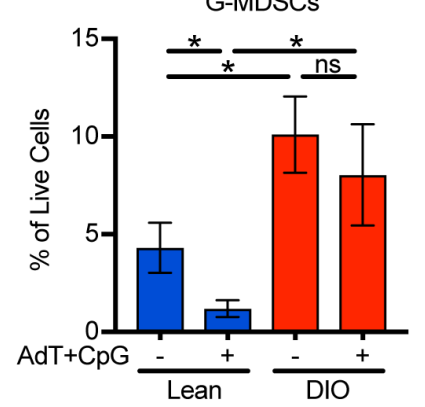

C

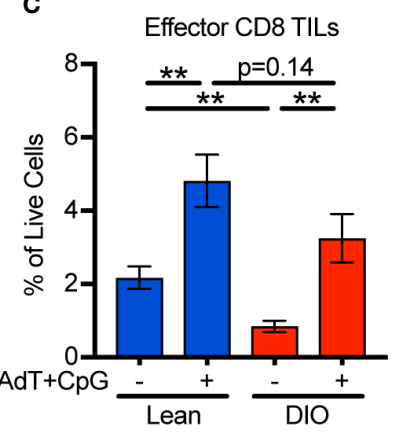

G

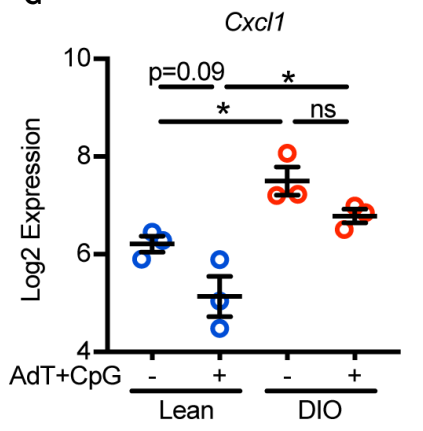

FIGURE 4 | Obesity promotes immunotherapy resistance and is associated with granulocytic myeloid-derived suppressor cell (G-MDSC) accumulation. Lean and DIO mice with established E0771-fluc tumors within a 25-100 mm² range were randomized to receive saline control or AdT+CpG immunotherapy. (A) Tumor areas at therapy initiation. (B) Resulting tumor growth for lean and DIO animals. Flow cytometric analysis of day 28 excised mammary tumors for (C) effector (CD44 $\left.{ }^{+} \mathrm{CD} 62 \mathrm{~L}^{-}\right)$and

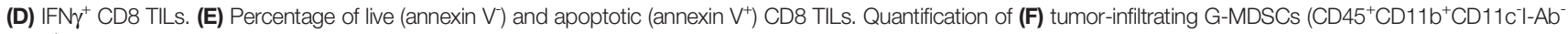
Ly6C ${ }^{\text {low }} \mathrm{Ly}_{6 \mathrm{G}}{ }^{+}$). (G) Log2 expression of $\mathrm{CxCl1}$ in the tumor microenvironment. (A-D,F) Data are pooled from multiple independent experiments ( $\mathrm{n}=6-21$ per group) or (E) are from single a representative experiment ( $n=4-9$ per group) and presented as means \pm SEM or (B) individual outgrowth is shown, with the bold line indicating mean tumor growth. Statistical differences were calculated using repeated measures two-way ANOVAs with Tukey's multiple comparison or non-parametric Kruskal-Wallis tests or parametric one-way ANOVAs with post hoc pairwise comparison of group means of interest using non-parametric Mann-Whitney $U$ tests, parametric two-tailed unpaired t-tests, or Tukey's multiple comparisons tests as appropriate $\left({ }^{\star} \mathrm{p}<0.05,{ }^{\star \star} \mathrm{p}<0.01,{ }^{\star \star \star} \mathrm{p}<0.001\right.$, ns $=$ non-significant).

immunotherapy resistance in obese animals, we targeted CXCR2, which is highly and predominantly expressed on GMDSCs (Figures 3D, 5A). Treatment of lean animals with combinatorial AdT $+\mathrm{CpG}+\mathrm{CXCR} 2$ antagonist (CXCR2a) provided no benefit over that of $\mathrm{AdT}+\mathrm{CpG}$ alone with regard to tumor burden or MDSC accumulation (Figures 5B-D). In fact, the combinatorial therapy reduced the proportion of lean animals responding to therapy (7/8 versus $4 / 9)$, indicating potential negative effects. Interestingly, no change was seen in G-MDSC accumulation with the addition of the CXCR2a, likely due to the substantial reduction caused by AdT+CpG alone.

Following therapy administration, few beneficial responses were evident in obese animals treated with the CXCR2a (1/5) or AdT $+\mathrm{CpG}$ (3/9) alone (Figure 5E). However, the combination of the CXCR2a and AdT+CpG led to substantially improved responses in the majority of animals treated (8/13) (Figure $5 \mathbf{E})$, corresponding with significant reductions in tumor burdens (Figure 5F) and G-MDSC accumulation (Figure 5G). Thus, the combinatorial AdT+CpG+CXCR2a immunotherapy was able to reduce G-MDSC accumulation and improve immunotherapy responses, specifically in obese animals.

\section{CXCL1 Expression in Breast Tumors Is Positively Associated With BMI, Poor Overall Survival, and a MDSC Expression Score}

Last, we evaluated the clinical relevance of our findings by utilizing previously published immunogenetic and transcriptomic data from tumor tissues of patients with breast cancer. We first used linear regression analyses to evaluate the association of body mass index (BMI), a metric used to define obesity, with the expression of 730 immune-related genes. In a cohort of 68 patients with triple negative breast cancer (17) 73 of 730 total genes were found to be associated with BMI $(\mathrm{p}<0.1)$ (Figure 6A). Of these 73 genes, 65 were negatively associated with BMI and 8 were positively associated with BMI. These 8 genes included CXCL1, S100A8, RELA, CCL20, CDK1, NOD2, SH2B2, and TANK (Figure 6A). GO assessment of biological processes associated with these 8 genes revealed neutrophil/granulocyte chemotaxis/migration, corroborating immunogenetic, protein, and cellular results from our murine study (Figure 6B). Importantly, CXCL1, S100A8, and NOD2 were also found to be positively associated with obesity in our murine study. Increased expression of CXCL1 was not only 

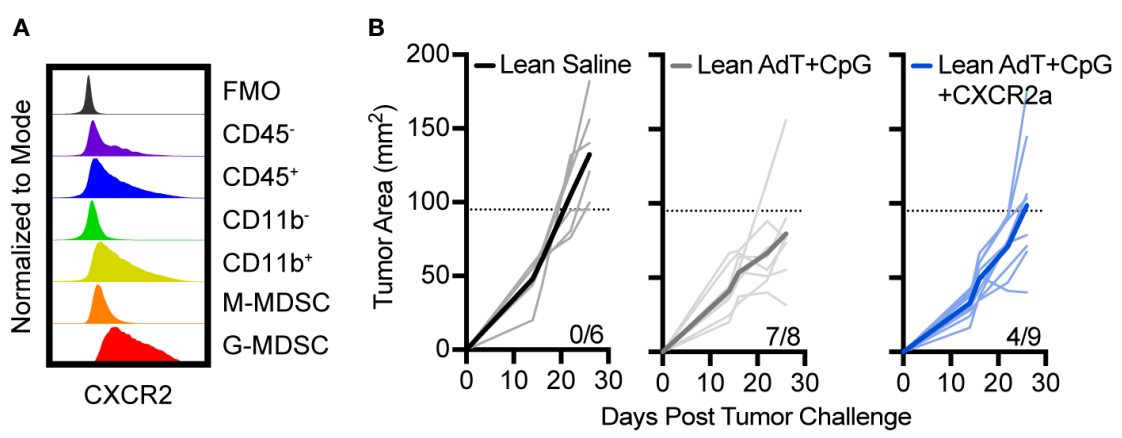
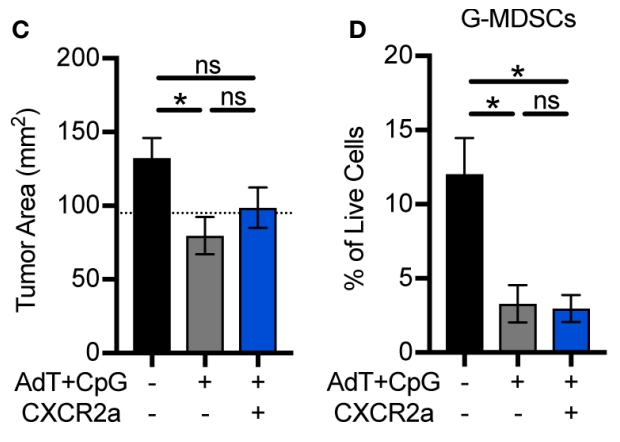

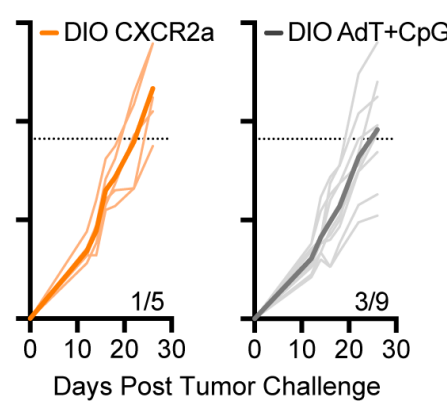

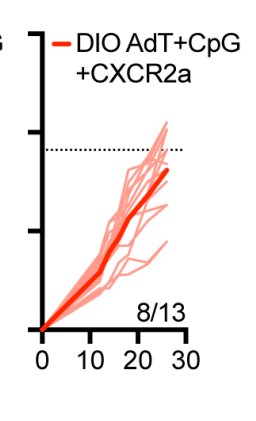
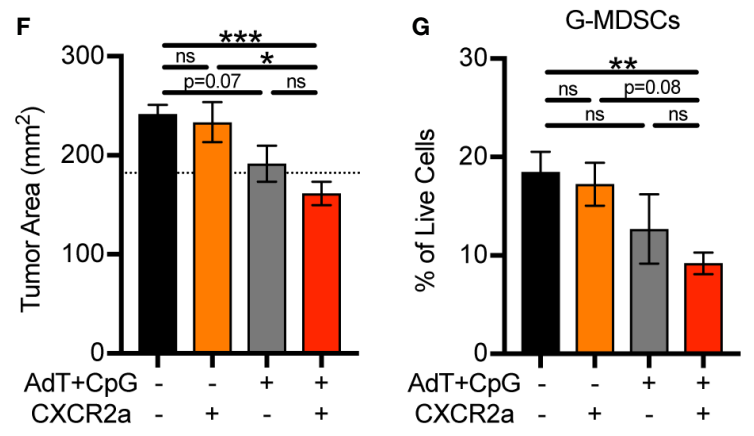

FIGURE 5 | Disruption of granulocytic myeloid-derived suppressor cells (G-MDSC) accumulation sensitizes obese animals to immunotherapy. (A) Representative histogram depicting intracellular expression of CXCR2 in different populations in the E0771-fluc tumor microenvironment. Lean and DIO animals bearing E0771-fluc tumors were treated with saline, CXCR2 antagonist (CXCR2a), AdT+CpG, or AdT+CpG+CXCR2a. Resulting tumor growth in (B) lean and (E) DIO animals is shown. Response thresholds are depicted by the dotted lines which indicate the lower limit of tumor growth in respective saline treated animals. Quantification of (C, F) endpoint tumor areas and (D, G) tumor-infiltrating G-MDSCs (CD45 ${ }^{+}$CD11 b ${ }^{+}$CD11 ${ }^{-}$I-Ab ${ }^{-}$Ly6C ${ }^{\text {low }}$ Ly6G $\left.^{+}\right)$. Data are pooled from multiple independent experiments and presented as means \pm SEM or individual outgrowth is shown, with the bold line indicating mean tumor growth. Statistical differences were calculated using parametric one-way ANOVAs with Tukey's multiple comparisons tests ( ${ }^{\star} p<0.05,{ }^{\star \star} p<0.01$, ${ }^{\star \star \star} p<0.001$, ns $=$ non-significant).

found to be associated with increasing BMI (Figure 6C), but also decreased overall survival in a cohort of $\mathrm{n}=54$ systemically untreated patients with basal breast cancer (Figure 6D). Analysis of transcriptomic data available from The Cancer Genome Atlas (TCGA) allowed us to quantify the relative abundance of MDSCs using a previously published multi-gene MDSC expression score (22). In breast tumor tissues from $n=88$ patients with basal breast cancer, CXCL1 and FASLG expression were found to increase significantly as the MDSC expression score increased (Figure 6E). These data support the clinical relevance of our murine findings by providing evidence that the expression levels of multiple MDSC chemokines (i.e., CXCL1 and S100A8) were positively associated with BMI in patients with breast cancer. High CXCL1 expression was further associated with poor overall survival and expressionbased MDSC abundance. Additionally, this MDSC score was positively associated with expression of FASLG.

\section{DISCUSSION}

The prevalence of obesity among adults in the US is currently approaching $40 \%$ (1) and is expected to reach $50 \%$ by 2030 (27). As many cancer patients are now being treated with immunotherapies, understanding the impact of obesity on antitumor immunity and immunotherapeutic outcomes is critical. Here, we report that, in a preclinical model of breast cancer, obesity impairs antitumor immunity and promotes immunotherapy resistance. Specifically, we have identified a novel pathway wherein obesity drives hyperactivation of and subsequent Fas expression on CD8 TILs, priming them for apoptosis. Concurrently, obesity increases intratumoral CXCL1 concentrations, promoting CXCR2-mediated accumulation of $\mathrm{FasL}^{+}$G-MDSCs, resulting in heightened Fas/FasL-mediated CD8 TIL apoptosis and immunotherapy resistance (Figure 7). We find evidence of this pathway in lean animals, albeit to a lesser extent (i.e. low levels of both FasL ${ }^{+}$G-MDSCs and CD8 TIL apoptosis). Thus we posit that the above mechanism is primarily operative in animals with obesity. The clinical relevance of this pathway is illustrated by our transcriptomic data showing high concordant expression of CXCL1 with increasing BMI, poor survival, and a MDSC expression score in human breast tumors.

Our data add to a growing number of reports detailing the complex role of obesity in modulating antitumor immunity and responses to immunotherapy. Recent reports indicate that obesity not only promotes CD8 T cell dysfunction in a preclinical model of melanoma (28), but also leads to reductions in CD8 TILs in murine and human breast tumors (6). In the latter study, TIL reductions occurred in parallel with increased apoptosis of CD $45^{+}$leukocytes, 
A

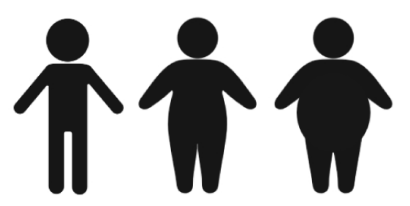

Breast tumor $(n=68)$ TNBC nanoString immunogenetic profiling
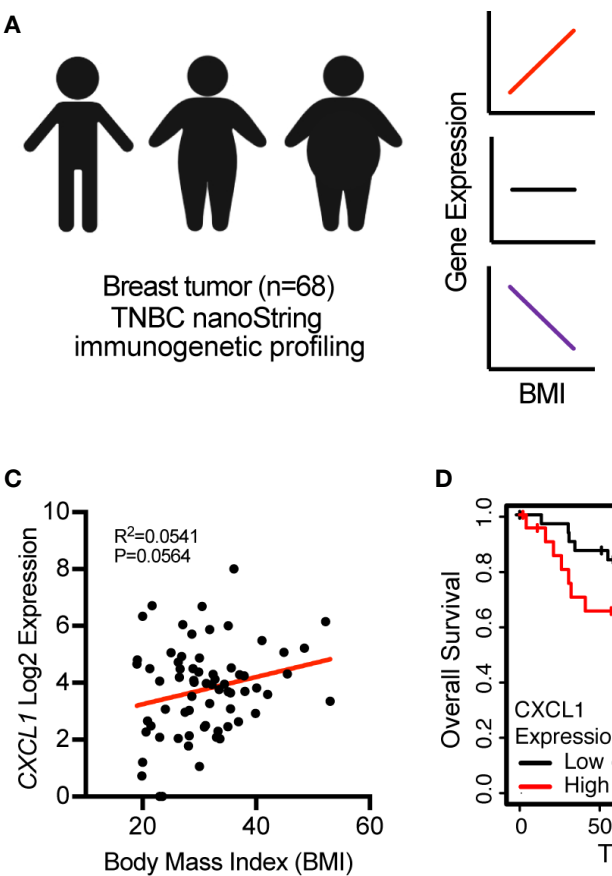

D

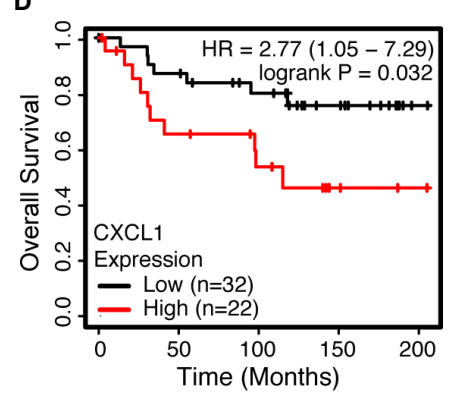

B

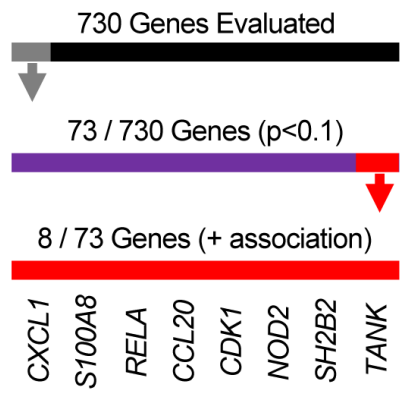

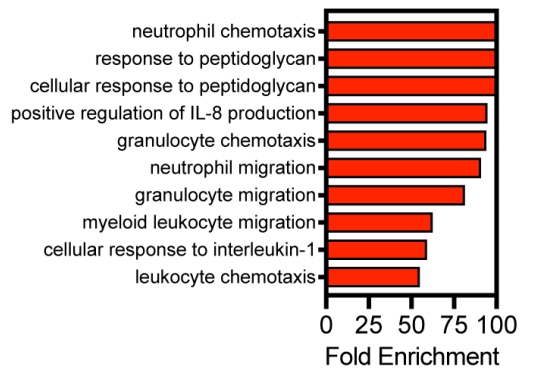

E

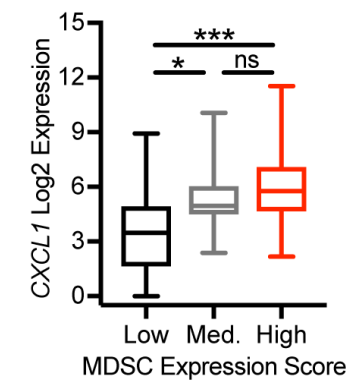

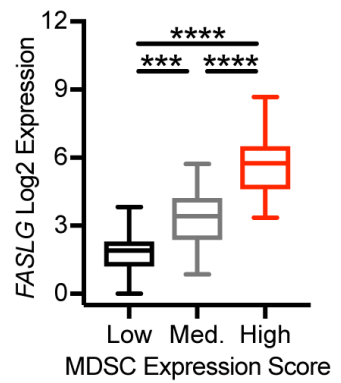

FIGURE 6 | CXCL1 expression in breast tumors is positively associated with BMI, poor overall survival, and a myeloid-derived suppressor cell (MDSC) expression score. (A) NanoString immunogenetic profiling of $n=68$ triple negative breast tumors. Of 730 genes surveyed, 73 were found to be associated ( $p<0.1)$ with body mass index (BMI). Of the 73 genes identified, 8/73 were positively associated with BMl and 65/73 were negatively associated with BMI. (B) Gene Ontology (GO)identified biological processes associated with the 8 indicated genes. (C) Correlation between BMI and CXCL1 expression from samples used in (A, B). (D) Expression of CXCL1 and overall survival in systemically untreated patients with basal breast cancer. Data were generated using KM Plotter ( $\mathrm{kmplot.com).}$ (E) The Cancer Genome Atlas (TCGA)-derived transcriptomics data from breast tumor tissue of $n=88$ patients with basal breast cancer. Multi-gene MDSC expression score was calculated and samples were separated into low $(n=20)$, medium $(n=20)$, and high $(n=48)$ tertiles. Analysis of myeloid-derived suppressor cells (MDSC) expression scores versus expression of (left) CXCL1 and (right) FASLG. Data are presented as linear regression, Kaplan-Meier plots, or boxes defining $25^{\text {th }}$ to $75^{\text {th }}$ percentiles with line at median and whiskers extending to minimum and maximum points. Statistical differences were calculated using linear regression, log rank test, or parametric one-way ANOVAs with Tukey's multiple comparisons tests $\left({ }^{\star} \mathrm{p}<0.05,{ }^{\star \star \star} \mathrm{p}<0.001,{ }^{\star \star \star \star} \mathrm{p}<0.0001\right)$.

although CD8 TIL apoptosis was not specifically examined. We add to this literature by demonstrating that obesity not only drives hyperactivation of CD8 TILs, but also predisposes them to GMDSC-mediated apoptosis, thereby impeding antitumor immunity and immunotherapeutic efficacy (29).

Obesity and inflammation expand and mobilize MDSCs and their precursors via molecules such as IL-6, CRP, IL-1 $\beta$, and leptin (13-16, 30), increasing their susceptibility to tumor-derived signals that further drive their recruitment and suppressive capacity. Here, we found that the expression levels of multiple mediators of MDSC development and chemotaxis, including Cxcl1, Cxcl3, S100a8, Csf3 (G-CSF), and Il1 $\beta$ (31), were elevated in the tumors of DIO animals (Figures 1E, 3B and Supplementary Table 1). These results were further supported by our clinical analyses which demonstrated positive associations between BMI and the expression of CXCL1 and S100A8 in human breast tumors (Figure 6). These data provide evidence for redundant mechanisms of MDSC recruitment to the tumor microenvironment in mice and humans with obesity, highlighting the need for further investigation of obesityassociated alterations in the soluble protein milieu of the tumor microenvironment. Here, we targeted CXCR2 for intervention in mice with mammary tumors, disrupting signals from its cognate ligands, CXCL1 and CXCL3. Although a significant therapeutic benefit was observed, chemotactic redundancy (i.e., S100A8) was likely responsible for achieving only a modest reduction in tumor burden. This redundancy may serve as a barrier for the clinical targeting of MDSCs, particularly in the context of obesity. Thus, interventions targeting MDSC generation or function, rather than chemotaxis, may be more efficacious in the clinical setting.

A recent study utilized single cell transcriptomics and the MMTV-PyMT transgenic murine model of breast cancer to identify expression signatures found in G-MDSCs versus their non-suppressive neutrophil counterparts (32). The authors identified genes, including Arg2, Cxcr2, Illb, Ifitm1, Socs3, Osm, and Cd14 among others, whose expression was elevated in G-MDSCs. These genes identify particular mechanisms of suppression (i.e., Arg2, which encodes arginase 2) and chemotaxis (i.e., Cxcr2) utilized by G-MDSCs $(25,33)$. In our study, all of the aforementioned genes were increased in expression in the tumors of obese mice (Supplementary Table 2). These data further support the classification of G-MDSCs in our murine model and emphasize the accumulation of GMDSCs rather than non-suppressive neutrophils.

Recently, tumor-infiltrating MDSCs have been shown to promote immunotherapy resistance in preclinical models of 


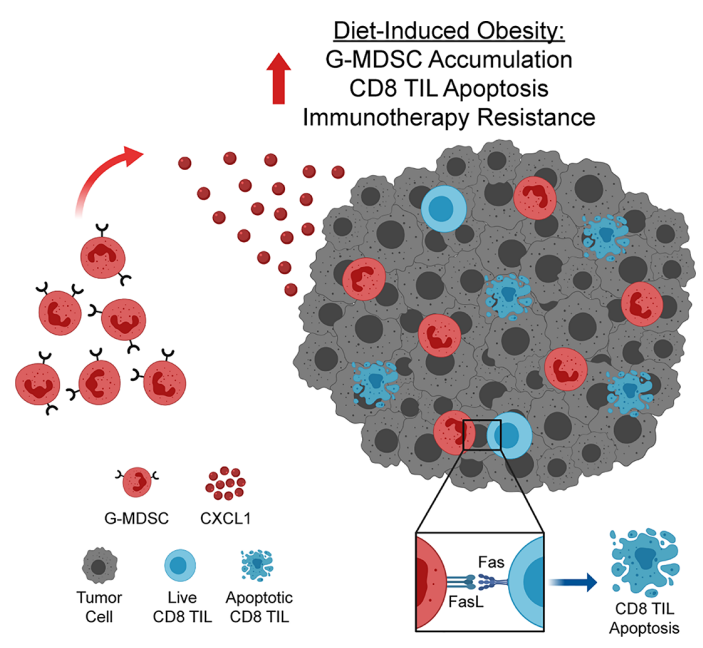

FIGURE 7 | Obesity enhances accumulation of $\mathrm{FasL}^{+}$granulocytic myeloidderived suppressor cells (G-MDSCs), driving CD8 TIL apoptosis and immunotherapy resistance. Increased concentration of CXCL1 in the obese mammary tumor microenvironment drives CXCL1/CXCR2-mediated accumulation of FasL ${ }^{+}$G-MDSCs. FasL ${ }^{+}$G-MDSCs interact with hyperactivated Fas ${ }^{+}$CD8 TILs, driving CD8 TIL apoptosis and immunotherapy resistance. Graphic created with BioRender.com.

breast cancer and melanoma $(17,18)$. In studies of inducible and transplantable melanoma, MDSC-mediated immunotherapy resistance was found to occur in part through expression of FasL and subsequent Fas/FasL-mediated apoptosis of CD8 TILs (18). Here, we substantiated those findings and extended them to breast cancer, where we implicate FasL-expressing MDSCs as part of a broader pathway that links host obesity to heightened CD8 TIL apoptosis and immunotherapy resistance. We find that targeting of MDSCs is sufficient to improve immunotherapy response, although the subsequent impact on CD8 TIL dynamics was not evaluated. Obesity is an incredibly complex and multifactorial disease that elicits numerous changes to the breast tumor microenvironment (34). As such, we recognize the potential for additional known, or currently unknown, factors to contribute to our observed outcomes.

Reports indicate that elevated levels of plasma IL-6 and CRP are associated with reduced progression free and overall survival in patients with breast cancer who were treated with anti-PD-L1 immunotherapy (12). Notably, both IL-6 and CRP are elevated with obesity and modulate MDSC dynamics $(15,16)$. Although the impact of obesity on immunotherapy outcomes in patients with breast cancer has not yet been evaluated, the aforementioned data suggest that obesity may be associated with worse clinical outcomes in breast cancer, which may be linked to MDSC accumulation. This notion is backed by our transcriptomic analyses, which shows a positive correlation between CXCL1 expression and BMI, poor survival, and a MDSC expression score in human breast tumor tissues (Figure 4E). Additional evidence for this hypothesis comes from earlier work implicating obesity in resistance to targeted and chemotherapies in patients with breast cancer $(7,8)$. Our preclinical findings concur with these outcomes. Conversely, a retrospective analysis of women with melanoma found that obesity did not impact outcomes to anti-PD-1/PD-L1 immunotherapy (35). As this study was retrospective, there was no evaluation of IL-6, CRP, or MDSCs, indicating areas for future prospective analyses. Thus, these data suggest obesity may be associated with worse immunotherapy outcomes in patients with breast cancer, but also that obesity may differentially impact treatment outcomes based on tumor or immunotherapy type, emphasizing the need for continued study of this critical issue.

With nearly $40 \%$ of the adult US population presenting with obesity and an additional $30 \%$ presenting with overweight (1), it is critical now more than ever that we consider co-morbidities, such as obesity and diabetes, as the new normal. This is especially needed in preclinical modeling where the use of young lean mice starkly departs from our aging and weighty cancer patient population. Forethought will allow us to more considerately and effectively develop therapeutics to treat what continues to be the increasing majority of our population.

\section{DATA AVAILABILITY STATEMENT}

The differentially expressed genes identified from the murine mammary tumor immunogenetic profiling results in this study can be found in Supplementary Table 2. Immunogenetic and transcriptomic profiling data from human breast tumors were obtained from a previously published manuscript (17) or TCGA and are publicly available.

\section{ETHICS STATEMENT}

All animal experiments were conducted as approved by the Animal Resources Program and Institutional Animal Care and Use Committee at the University of Alabama at Birmingham (UAB).

\section{AUTHOR CONTRIBUTIONS}

JG and LN conceptualized the study, designed experiments, and wrote the manuscript. JG managed and led the study under the supervision of LN. JG, RO, WT, and MB performed experiments and analyzed data. All authors contributed to the article and approved the submitted version.

\section{FUNDING}

Research reported in this publication was supported by the University of Alabama at Birmingham Department of Nutrition Sciences startup package to LN, Breast Cancer Research Foundation of Alabama Impact Grant to LN, NIH award \#T32GM008111 to JG, NCI award \#T32CA047888 to RO and MB, NCI award \#T32CA183926 to WT, the Rita Allen Foundation grant 
\#2014701 to RS, and NIH awards \#P30CA013148 and \#1S10OD021697 to the UAB O’Neal Comprehensive Cancer Center Preclinical Imaging Shared Facility.

\section{ACKNOWLEDGMENTS}

The authors would like to thank the following UAB cores and facilities: nanoString laboratory, particularly Debbie Della Manna, flow cytometry core, particularly Vidya Sagar Hanumanthu and Berencia M Fore, and the O'Neal Comprehensive Cancer Center Preclinical Imaging Shared Facility. We would also like to thank

\section{REFERENCES}

1. Hales CM, Fryar CD, Carroll MD, Freedman DS, Ogden CL. Trends in Obesity and Severe Obesity Prevalence in US Youth and Adults by Sex and Age, 20072008 to 2015-2016. JAMA (2018) 319(16):1723-5. doi: 10.1001/jama.2018.3060

2. Lauby-Secretan B, Scoccianti C, Loomis D, Grosse Y, Bianchini F, Straif K, et al. Body Fatness and Cancer-Viewpoint of the IARC Working Group. N Engl J Med (2016) 375(8):794-8. doi: 10.1056/NEJMsr1606602

3. Kolb R, Phan L, Borcherding N, Liu Y, Yuan F, Janowski AM, et al. Obesityassociated NLRC4 inflammasome activation drives breast cancer progression. Nat Commun (2016) 7:13007. doi: 10.1038/ncomms13007

4. Quail DF, Olson OC, Bhardwaj P, Walsh LA, Akkari L, Quick ML, et al. Obesity alters the lung myeloid cell landscape to enhance breast cancer metastasis through IL5 and GM-CSF. Nat Cell Biol (2017) 19(8):974-87. doi: $10.1038 / \mathrm{ncb} 3578$

5. Michelet X, Dyck L, Hogan A, Loftus RM, Duquette D, Wei K, et al. Metabolic reprogramming of natural killer cells in obesity limits antitumor responses. Nat Immunol (2018) 19(12):1330-40. doi: 10.1038/s41590-018-0251-7

6. Zhang C, Yue C, Herrmann A, Song J, Egelston C, Wang T, et al. STAT3 Activation-Induced Fatty Acid Oxidation in CD8(+) T Effector Cells Is Critical for Obesity-Promoted Breast Tumor Growth. Cell Metab (2020) 31 (1):148-61.e5. doi: 10.1016/j.cmet.2019.10.013

7. Greenlee H, Unger JM, LeBlanc M, Ramsey S, Hershman DL. Association between Body Mass Index and Cancer Survival in a Pooled Analysis of 22 Clinical Trials. Cancer Epidemiol Biomarkers Prev (2017) 26(1):21-9. doi: 10.1158/1055-9965.EPI-15-1336

8. Incio J, Ligibel JA, McManus DT, Suboj P, Jung K, Kawaguchi K, et al. Obesity promotes resistance to anti-VEGF therapy in breast cancer by up-regulating IL-6 and potentially FGF-2. Sci Transl Med (2018) 10(432):eaag0945. doi: 10.1126/scitranslmed.aag0945

9. Chan DS, Vieira AR, Aune D, Bandera EV, Greenwood DC, McTiernan A, et al. Body mass index and survival in women with breast cancer-systematic literature review and meta-analysis of 82 follow-up studies. Ann Oncol (2014) 25(10):1901-14. doi: 10.1093/annonc/mdu042

10. Caan BJ, Cespedes Feliciano EM, Prado CM, Alexeeff S, Kroenke CH, Bradshaw P, et al. Association of Muscle and Adiposity Measured by Computed Tomography With Survival in Patients With Nonmetastatic Breast Cancer. JAMA Oncol (2018) 4(6):798-804. doi: 10.1001/jamaoncol.2018.0137

11. Schmid P, Adams S, Rugo HS, Schneeweiss A, Barrios CH, Iwata H, et al. Atezolizumab and Nab-Paclitaxel in Advanced Triple-Negative Breast Cancer. N Engl J Med (2018) 379(22):2108-21. doi: 10.1056/NEJMoa1809615

12. Li Y, Fassò M, Emens LA, Molinero L. Abstract CT001: Biomarkers of systemic inflammation associated to reduced clinical activity of atezolizumab monotherapy in patients with metastatic triple negative breast cancer. Cancer Res (2019) 79(13 Supplement):CT001-CT. doi: 10.1158/15387445.AM2019-CT001

13. Bunt SK, Sinha P, Clements VK, Leips J, Ostrand-Rosenberg S. Inflammation induces myeloid-derived suppressor cells that facilitate tumor progression. J Immunol (2006) 176(1):284-90. doi: 10.4049/jimmunol.176.1.284

14. Clements VK, Long T, Long R, Figley C, Smith DMC, Ostrand-Rosenberg S. Frontline Science: High fat diet and leptin promote tumor progression by
Brett P. Gross for generation of the firefly luciferase expressing variant of the E0771 cell line, Dr. Francis E. Lund for use of her ab's MAGPIX Luminex plate reader, Dr. Davide Botta for running the multiplex immunoassays, and Dr. Alexander Szalai and Dr. Douglas Hurst for reading and providing feedback on the manuscript.

\section{SUPPLEMENTARY MATERIAL}

The Supplementary Material for this article can be found online at: https://www.frontiersin.org/articles/10.3389/fimmu.2020. 590794/full\#supplementary-material

inducing myeloid-derived suppressor cells. J Leukoc Biol (2018) 103(3):395407. doi: 10.1002/JLB.4HI0517-210R

15. Jimenez RV, Kuznetsova V, Connelly AN, Hel Z, Szalai AJ. C-Reactive Protein Promotes the Expansion of Myeloid Derived Cells With Suppressor Functions. Front Immunol (2019) 10:2183. doi: 10.3389/fimmu.2019.02183

16. Marigo I, Bosio E, Solito S, Mesa C, Fernandez A, Dolcetti L, et al. Tumorinduced tolerance and immune suppression depend on the C/EBPbeta transcription factor. Immunity (2010) 32(6):790-802. doi: 10.1016/ j.immuni.2010.05.010

17. Kim IS, Gao Y, Welte T, Wang H, Liu J, Janghorban M, et al. Immunosubtyping of breast cancer reveals distinct myeloid cell profiles and immunotherapy resistance mechanisms. Nat Cell Biol (2019) 21(9):1113-26. doi: 10.1038/s41556-019-0373-7

18. Zhu J, Powis de Tenbossche CG, Cane S, Colau D, van Baren N, Lurquin C, et al. Resistance to cancer immunotherapy mediated by apoptosis of tumorinfiltrating lymphocytes. Nat Commun (2017) 8(1):1404. doi: 10.1038/s41467017-00784-1

19. Mi H, Muruganujan A, Ebert D, Huang X, Thomas PD. PANTHER version 14: more genomes, a new PANTHER GO-slim and improvements in enrichment analysis tools. Nucleic Acids Res (2019) 47(D1):D419-D26. doi: 10.1093/nar/gky1038

20. Norian LA, Kresowik TP, Rosevear HM, James BR, Rosean TR, Lightfoot AJ, et al. Eradication of metastatic renal cell carcinoma after adenovirus-encoded TNF-related apoptosis-inducing ligand (TRAIL)/CpG immunotherapy. PLoS One (2012) 7(2):e31085. doi: 10.1371/journal.pone.0031085

21. Gyorffy B, Lanczky A, Eklund AC, Denkert C, Budczies J, Li Q, et al. An online survival analysis tool to rapidly assess the effect of 22,277 genes on breast cancer prognosis using microarray data of 1,809 patients. Breast Cancer Res Treat (2010) 123(3):725-31. doi: 10.1007/s10549-009-0674-9

22. Wang G, Lu X, Dey P, Deng P, Wu CC, Jiang S, et al. Targeting YAPDependent MDSC Infiltration Impairs Tumor Progression. Cancer Discov (2016) 6(1):80-95. doi: 10.1158/2159-8290.CD-15-0224

23. Ali HR, Provenzano E, Dawson SJ, Blows FM, Liu B, Shah M, et al. Association between CD8+ T-cell infiltration and breast cancer survival in 12,439 patients. Ann Oncol (2014) 25(8):1536-43. doi: 10.1093/annonc/mdu191

24. Dominguez GA, Condamine T, Mony S, Hashimoto A, Wang F, Liu Q, et al. Selective Targeting of Myeloid-Derived Suppressor Cells in Cancer Patients Using DS-8273a, an Agonistic TRAIL-R2 Antibody. Clin Cancer Res (2017) 23 (12):2942-50. doi: 10.1158/1078-0432.CCR-16-1784

25. Griffith JW, Sokol CL, Luster AD. Chemokines and chemokine receptors: positioning cells for host defense and immunity. Annu Rev Immunol (2014) 32:659-702. doi: 10.1146/annurev-immunol-032713-120145

26. Zoglmeier C, Bauer H, Noerenberg D, Wedekind G, Bittner P, Sandholzer N, et al. CpG blocks immunosuppression by myeloid-derived suppressor cells in tumor-bearing mice. Clin Cancer Res (2011) 17(7):1765-75. doi: 10.1158/ 1078-0432.CCR-10-2672

27. Ward ZJ, Bleich SN, Cradock AL, Barrett JL, Giles CM, Flax C, et al. Projected U.S. State-Level Prevalence of Adult Obesity and Severe Obesity. N Engl J Med (2019) 381(25):2440-50. doi: 10.1056/NEJMsa1909301

28. Wang Z, Aguilar EG, Luna JI, Dunai C, Khuat LT, Le CT, et al. Paradoxical effects of obesity on $\mathrm{T}$ cell function during tumor progression and PD-1 
checkpoint blockade. Nat Med (2019) 25(1):141-51. doi: 10.1038/s41591-0180221-5

29. Horton BL, Williams JB, Cabanov A, Spranger S, Gajewski TF. Intratumoral CD8(+) T-cell Apoptosis Is a Major Component of T-cell Dysfunction and Impedes Antitumor Immunity. Cancer Immunol Res (2018) 6(1):14-24. doi: 10.1158/2326-6066.CIR-17-0249

30. Nagareddy PR, Kraakman M, Masters SL, Stirzaker RA, Gorman DJ, Grant $\mathrm{RW}$, et al. Adipose tissue macrophages promote myelopoiesis and monocytosis in obesity. Cell Metab (2014) 19(5):821-35. doi: 10.1016/ j.cmet.2014.03.029

31. Umansky V, Blattner C, Gebhardt C, Utikal J. The Role of Myeloid-Derived Suppressor Cells (MDSC) in Cancer Progression. Vaccines (Basel) (2016) 4 (4):36. doi: 10.3390/vaccines4040036

32. Alshetaiwi H, Pervolarakis N, McIntyre LL, Ma D, Nguyen Q, Rath JA, et al. Defining the emergence of myeloid-derived suppressor cells in breast cancer using single-cell transcriptomics. Sci Immunol (2020) 5(44):eaay6017. doi: 10.1126/sciimmunol.aay6017

33. Grzywa TM, Sosnowska A, Matryba P, Rydzynska Z, Jasinski M, Nowis D, et al. Myeloid Cell-Derived Arginase in Cancer Immune Response. Front Immunol (2020) 11:938. doi: 10.3389/fimmu.2020.00938
34. Gilbert CA, Slingerland JM. Cytokines, obesity, and cancer: new insights on mechanisms linking obesity to cancer risk and progression. Annu Rev Med (2013) 64:45-57. doi: 10.1146/annurev-med-121211-091527

35. McQuade JL, Daniel CR, Hess KR, Mak C, Wang DY, Rai RR, et al. Association of body-mass index and outcomes in patients with metastatic melanoma treated with targeted therapy, immunotherapy, or chemotherapy: a retrospective, multicohort analysis. Lancet Oncol (2018) 19(3):310-22. doi: 10.1016/S1470-2045(18)30078-0

Conflict of Interest: The authors declare that the research was conducted in the absence of any commercial or financial relationships that could be construed as a potential conflict of interest.

Copyright $\odot 2020$ Gibson, Orlandella, Turbitt, Behring, Manne, Sorge and Norian. This is an open-access article distributed under the terms of the Creative Commons Attribution License (CC BY). The use, distribution or reproduction in other forums is permitted, provided the original author(s) and the copyright owner(s) are credited and that the original publication in this journal is cited, in accordance with accepted academic practice. No use, distribution or reproduction is permitted which does not comply with these terms. 